\title{
Measuring the effectiveness of psychoeducation on adherence, depression, anxiety and stress among patients with diagnosis of schizophrenia. a control trial
}

\author{
Apostolia Alizioti $^{1,2}$ (D) $\cdot$ Georgios Lyrakos ${ }^{2,3}$ \\ Published online: 5 June 2019 \\ (C) The Author(s) 2019
}

\begin{abstract}
Despite evidence of the impact of depression and stress on attitudes towards medication in general medical pathology regimens, little is known of the effects of depression and stress on attitudes towards medication in schizophrenia. This study explores the effects of a hope-based psychoeducation on attitudes towards medication, depression, anxiety and stress, and predictors of attitudes towards medication. The participants in the study $(n=29)$ were randomly allocated to either the psychoeducation group or the control group. Results from the psychoeducation group revealed that attitudes towards medication were significantly improved and anxiety was significantly reduced. Given the small sample, which was recruited from among patients of a day mental health centre, findings should be interpreted tentatively. Being longer in pharmacotherapy, having experienced fewer occurrences of hospitalisation, and being less depressed predicted positivity towards medication. Patients in the control group did not exhibit significant change in any of the studied variables. Findings were interpreted in the light of research on stigma and insight into illness, and add modestly to literature arguing for the importance of patients' retention of hope, empowerment and sense of control over illness. This study proposed that psychoeducation is an appropriate intervention to address a wide range of factors that compound adherence to medication and patients' symptoms, such as patients' interpretations of causal models, their sense of hope and control over the illness, and their insight into illness and self-stigma.
\end{abstract}

Keywords Schizophrenia $\cdot$ Adherence $\cdot$ Depression $\cdot$ Anxiety $\cdot$ Hope $\cdot$ Stigma $\cdot$ Insight

Research into schizophrenia suggests that the sudden disruption of neuroleptic drugs and non-adherence to taking them are associated with frequency of relapses, increased hospitalisation, suicidality (Haddad et al. 2014; Higashi et al. 2013), and mortality (Cullen et al. 2013). Medication adherence has been defined as the extent to which medication intake is consistent with medical prescriptions (Jimmy and Jose 2011). Patients are considered adherent to the treatment when

Electronic supplementary material The online version of this article (https://doi.org/10.1007/s12144-019-00255-4) contains supplementary material, which is available to authorized users.

Apostolia Alizioti

aliziotiapostolia@gmail.com

1 Cardiff School of Health Sciences, Cardiff Metropolitan University, Cardiff, UK

2 Department of Psychology, City Unity College, Athens, Greece

3 Department of Psychiatry, General Hospital of Nikaia, Piraeus, Greece their intake ranges from 70 to $80 \%$ of the prescribed medication (Kane et al. 2013). However, a study that included 11,797 chronic psychiatric patients estimates that $72.1 \%$ discontinued their medication for at least 30 days and at least once during a period of one year (Conti et al. 2012).

In schizophrenia, depression and negative symptomatology are found to be both a significant (Kane et al. 2013; Lyrakos et al. 2014; Na et al. 2015) and a non-significant predictor of patients' attitudes towards medication (Steger et al. 2012; Yang et al. 2012). Contrastingly, depression is always a predictor of reduced motivation and negative attitudes towards medication among patients in the general medical pathology regimens (DiMatteo et al. 2000; Gonzalez et al. 2008; Grenard et al. 2011). For example, the meta-analysis by DiMatteo et al. (2000) - with 122 included studies on adherence in general medical conditions - reports apparent consistency across findings for the reverse association between depression and adherence.

Despite the fact that research consistently reports that depression decreases adherence to medication of patients with chronic illness (non-psychiatric), the findings from 
schizophrenia research are mixed (Beck et al. 2011; Sapra et al. 2008). In schizophrenia, depression might increase adherence to medication instead of decreasing it (Fleischhacker et al. 2003). A possible explanation would be that patients who have improved depressive symptomatology might be more inclined to underestimate the benefits of antipsychotic pharmacotherapy and decrease adherence to medication as they start feeling better (Santone et al. 2008). Moreover, adherence leads to more medication intake; therefore, it is probable that side effects increase as well, including increased depressive symptomatology due to medication (Fleischhacker et al. 2003; Higashi et al. 2013; Staring et al. 2009). However, some patients, despite low tolerance to medication and medication - related symptoms of depression might continue to be highly adherent to pharmacotherapy (Staring et al. 2009) due to good treatment response of psychotic symptoms and the wish of patients to be free of positive symptoms (Beck et al. 2011).

An alternative explanation would be that patients with schizophrenia, who suffer from depressive symptoms, can similarly to non-psychiatric patients be negatively influenced in terms of motivation and might similarly exhibit passivity because of depression (Fleischhacker et al. 2003). However, passivity in depressed patients with schizophrenia is often qualitatively different from passivity in depression related to chronic non-psychiatric illness. For example, a Random Control Trial (RCT) study (Lyrakos et al. 2012), suggests that despite the fact that the level of motivation and level of activity were similarly low among psychiatric patients and patients with diabetes mellitus, the level of mental fatigue and general fatigue of psychiatric patients was significantly worse. This finding highlights how observed motivation - and by extension the level of observed withdrawal - is qualitatively different in psychiatric patients involving significantly higher levels of mental and physical fatigue. Consequently, these qualitative differences in the experience of depression by psychiatric and non - psychiatric patients (Lyrakos et al. 2012) might explain to some extent the contradictory findings in schizophrenia research on the relationship between depression and adherence to medication. In schizophrenia, comorbid depression can be much more severe, accompanied by negative symptoms such as avolition (severely diminished self initiated activities including lack of social interaction or avoidance of social interaction) and alogia (deteriorated fluency and efficiency of thought) (Rector et al. 2005). In the occurrence of such symptoms, patients with schizophrenia might accept the prescribed medication without much discussion or resistance (Fleischhacker et al. 2003; Schennach-Wolff et al. 2009) and adhere to medication despite being depressed (Fleischhacker et al. 2003). For example, the findings of a study suggest that patients with schizophrenia who have depression or negative symptoms are more likely to be passively compliant (Sapra et al. 2008). Specifically, Sapra and colleagues, (Sapra et al. 2008) report that depression was a strong predictor of patients' being influenced by other people in respect of good adherence to medication, and that negative symptoms strongly predicted patients' higher felt-impact of authority. Similarly, Beck and colleagues, (Beck et al. 2011) report that patients with schizophrenia, who exhibit increased social influence and dependence on others' opinions for decision making (in regard to adherence), exhibit the most positive attitudes towards medication but also experience the highest levels of depression (Beck et al. 2011).

In parallel, higher depression, higher anxiety (Ward 2014) and negative attitudes towards medication are associated with stigma (Santone et al. 2008). The stigma pertaining to schizophrenia is possibly the worst compared to other illnesses (Ward 2014) and stigma - related behaviour, such as avoidance behaviour, avoidance of activities, avoidance of social contact and social withdrawal, is particularly difficult to distinguish from depression (Kleim et al. 2008). Also, the internalised stigma might lead to negative attitudes toward medication compliance because the act of taking medication is considered proof of the individual's mental illness (Roberts and Wolfson 2004). Moreover, stigma interacts with non-adherence in a different mode, through self-efficacy. Some patients with mental illness suffer from self-stigma, endorsing and internalising others' discriminatory perceptions related to mental illness, which has a range of negative effects on patients' cognition and behaviour, including low self-esteem and low self-efficacy (Corrigan and Rao 2012). Low self-efficacy is associated in turn with increased depression and decreased adherence (Kleim et al. 2008; Lysaker et al. 2007), more similar to the case of nonadherence in the general medical conditions ( $\mathrm{Li}$ et al. 2011; Martos-Mendez 2015). Birchwood and colleagues (Birchwood et al. 1993) suggest that patients' depression is related to schizophrenia diagnosis acceptance and patients' perceived low sense of control over the illness. More often than not, the diagnosis is accompanied by information for the biogenetic causal models of the disorder (Lüllmann et al. 2011). Although the promotion of biogenetic - as opposed to psychosocial - causal models seems to contribute positively to medication adherence, it also seems that acceptance of biogenetic attributions is associated with actual stigma, patients' selfstigma and passive adherence to treatment (Lüllmann et al. 2011; Read et al. 2006). In addition, there is evidence that non-acceptance of self-stigma and in particular patients' selfesteem, self-efficacy, capacity to hope for the future and to reconstruct a sense of self and self-agency are essential for good prognosis and outcome (Rector et al. 2005; Roberts and Wolfson 2004). Based on the findings of these studies, it is suggested that the communication of biogenetic causal models has a role in the development and perpetuation of patients' selfstigma and actual social stigma (Lüllmann et al. 2011).

Research estimates that more than $70 \%$ of patients with schizophrenia suffer from symptoms of depression, while the estimation of anxiety symptoms (although anxiety is 
highly prevalent) is complicated due to the nature of the disorder (Eisner et al. 2013). The profound role of depression, stress and anxiety in psychosis is well documented in research; however, they often remain untreated (Eisner et al. 2013). Also, these symptoms constitute the primary early signs of relapse into episodes and often persist in remission phase, deteriorating the illness course and patients' overall quality of life (Huppert et al. 2001; Üçok et al. 2006). Similar to depressive symptoms, stress complicates the impact of adherence factors on behaviour. A fair amount of research suggests that stress significantly decreases adherence in chronic illness (WHO 2003), such as in diabetes (Farrell et al. 2004), among HIV-positive patients under antiretroviral therapy (Bottonari et al. 2005) and patients who receive immunosuppression drugs (de Brito et al. 2016).

\section{Rationale of the Study: Aims and Hypothesis}

To the best of our knowledge there are no studies on the relationship between anxiety, or stress and attitudes towards medication in mental disorders or schizophrenia research, while there are a few studies with reported outcome concerning the relationship between depression and adherence, with mixed results (Beck et al. 2011; Kane et al. 2013; Kleim et al. 2008; Lyrakos et al. 2014; Na et al. 2015; Perkins et al. 2006; Perkins et al. 2008; Santone et al. 2008; Sapra et al. 2008; Steger et al. 2012; Yang et al. 2012). In addition, more research is needed for the exploration of the effect of psychoeducation on depression, distress and adherence in schizophrenia (Xia et al. 2011). Since psychoeducational interventions are in the form of seminars, they can contextually incorporate and tackle - in little time and at low cost - the earlier discussed heterogeneous issues related to schizophrenia (Baüml et al. 2006) that compound depression and attitudes towards medication. Meta-analytic research evidence suggests that psychoeducational interventions significantly contribute to a range of health behaviours, adherence or attitudes towards medication (Gonzalez-Soria et al. 2016; Xia et al. 2011), specifically in the reduction of fear to treatment and an improvement in patients' confidence in treatment (Hornung et al. 1998), as well as an overall improvement in patients' quality of life (Atkinson et al. 1996; Browne et al. 1996; Herz et al. 2000). Successively, such beneficial contributions have a positive influence on important health outcomes, the reduction in relapse rates (Lincoln et al. 2007a, 2007b) and the improvement in patients' overall quality of life (Browne et al. 1996).

This study proposes that the aim to empower patients with psychoeducation can be strengthened if its scope includes: a. the enhancement of patients' sense of hope for the future course of the illness (Giráldez et al. 2011; Lysaker and Buck 2008; Roberts and Wolfson 2004) and b. the strengthening of patients' sense of control over the illness (Birchwood et al. 1993). Hope is a structural ingredient for improvement of clinical symptoms (Schrank et al. 2008), for the outcome and for recovery from schizophrenia illness (Bonney and Stickley 2008), and is suggested as a basis for treatment in NICE clinical guidelines (NICE 2014). Information for the disorder and communication of genetic and biological causal models should ensure that it will not foster patients' passive illness behaviour and will not obstruct retention of hope (Lüllmann et al. 2011). A hope-based psychoeducation could stress the importance for patients to be more active, to be more focused on the management of depression, anxiety and stress and to be less passive towards the illness, enhancing in parallel a sense of controllability over the illness. In addition, psychoeducation could be beneficial in helping patients with schizophrenia to de-stigmatise medication treatment, while at the same time helping them to raise insight into illness.

This study hypothesised that a hope-based psychoeducational intervention (presented in Supplementary material) could possibly contribute to an improvement of patients' attitudes towards medication, as well as an alleviation of patients' depression, stress and anxiety. In particular, it is hypothesised that after psychoeducation attitudes towards medication would be improved and also, depression, anxiety and stress would be found to be reduced at post-measurements and significant predictors of patients' attitudes towards medication.

\section{Method}

This was a quasi-experimental pilot study. The study protocol was approved by Cardiff Metropolitan University, UK, ethics committee. The guidelines of the template for intervention description and replication (TIDieR; Hoffmann 2014) were followed where applicable.

\section{Participants and Setting}

Included were outpatients, who registered as members of a day mental health centre, any time between January 2013 December 2016 and). In total, seventy-one individuals were screened for inclusion and exclusion criteria. Inclusion criteria were: (a) outpatients; (b) aged older than 23; and (c) living with family; (d) with a diagnosis of schizophrenia (given before the patients' registration at the day centre). Patients, who were in acute condition or residents of mental health hostels or supported flats, were excluded from the study (10 individuals) for reasons of sample homogeneity. Sample homogeneity was preferred in order to eliminate the probability to miss differences between our study groups possibly due to sample heterogeneity. Of the 71 patients screened for inclusion criteria 61 were invited to participate in the study. All invited patients 
were briefed on the procedures of both study groups upfront. In total, 29 individuals accepted to participate, and after the completion of the recruiting process they were randomly allocated to either the psychoeducation or the control group. The in-person briefing, questionnaires' collection and the psychoeducational sessions took place at the premises of the day mental health centre.

\section{Procedure}

Informed consent was obtained from all individual participants included in the study. Participation was voluntary; no financial or other incentives were given. Attitudes towards medication, depression, anxiety and stress were measured before (December, 2016) and after (February, 2017) the psychoeducation with self-administered questionnaires.

Study Groups Both the psychoeducation and the control group consisted of attendants at the day mental health centre. On a weekly basis, the centre's program consists of 15 therapeutic and recreational groups, including group psychotherapy, occupational therapy, dialogue group, sports, painting, photography seminars, cooking and team meals. None of the participants of the intervention or the control group were in psychotherapy outside the day mental health centre. The control group received no intervention additional to the day centre's program. Figure 1 illustrates the participants' flow chart.

The Intervention Group The intervention group was given a leaflet with basic information about schizophrenia and a 4session psychoeducation cycle. The psychoeducation was implemented with the scope to: A. induce a sense of hope for the future; and B. enhance a sense of control over the disorder. The objectives and the structure of the hope-based psychoeducation are available in Supplementary material. Information was structured in order to help patients to develop a more energetic personal stance, to improve communication of upcoming illness issues with their psychiatrist, to optimise their participation in treatment decisions, and develop more positive attitudes towards medication. The content of the sessions aimed to detach the stigma from medication intake, to help patients disinvest in passivity-creating myths around psychosis, as well as to motivate patients to invest in the positive effects of medication and in small health behaviour changes (in terms of diet habits and physical activity that may ease symptoms of anxiety and depression). Participants who were allocated to the intervention group were also given a 5-page leaflet about schizophrenia to take home. The leaflet was written by the researchers (not validated) in plain language to make it easy for all patients to understand the content and it was a brief summary of the information obtained during the literature review and used in the psychoeducational intervention (please refer to Supplementray material, where the studies that were used for the production of the psychoeducational seminars are cited in the text). In particular, the leaflet gives basic information about symptoms and misconceptions in psychosis, the benefits of medication, early signs of relapse and about the role of physical activity and diet on stress management and depression. The sessions lasted approximately 60 min, including discussion and took place on 9,23 and 30, January and 6 February, 2017.

\section{Measures}

The Drug Attitudes Inventory (DAI) was originally developed for the assessment of actual adherence to medication among patients with schizophrenia (Hogan et al. 1983). The short version of DAI is a 10-item self-administered instrument with good internal consistency $(\mathrm{a}=0.81)$ and high test-retest reliability $(r=0.83)$, (Hogan et al. 1983). The items are in True/ False format. Six of the 10 items denote better adherence to medication if answered as "True", and the remaining four items indicate worse adherence to medication if answered as "True"; scored as plus one and minus one, accordingly. Attitudes towards medication were categorised into negative (low, medium, high), neutral and positive (low, medium and high).

The short version of Depression, Anxiety and Stress Scale (DASS-21), is made up of 21 self-report items. The scale has good internal consistency $(\alpha=0.93)$ and subscale coefficient alpha (depression $\alpha=0.83$, anxiety $\alpha=0.80$, stress $\alpha=0.89$ ) (Lovibond and Lovibond 1995; Lyrakos et al. 2011). In DASS, respondents are instructed to indicate how much each statement would apply to them within the last week. Each of the three subscales is assessed by seven questions of a fourpoint Likert-type scale, from 0 (did not apply to me at all) to 3 (applied to me very much or most of the time) and form four symptom-severity categories (mild, moderate, severe and extremely severe); with higher scores signifying greater severity.

A self-report general questionnaire with eight items was developed to record demographic information (sex, age, family status and number of people in the household), as well as basic clinical history information (years of medication treatment and occurrences of hospitalisation).

\section{Results}

Of the 29 recruited patients 27 completed the post measurements. From the psychoeducation group one patient was excluded due to failure to attend any session. Two-thirds of the patients in the intervention group attended three sessions or four sessions (six patients or four patients, respectively) and one-third of the patients attended only two sessions or one session (two patients in each case). 
Fig. 1 Flow diagram of study sample. The diagram shows the included patients, recruited patients, group allocation and drop outs

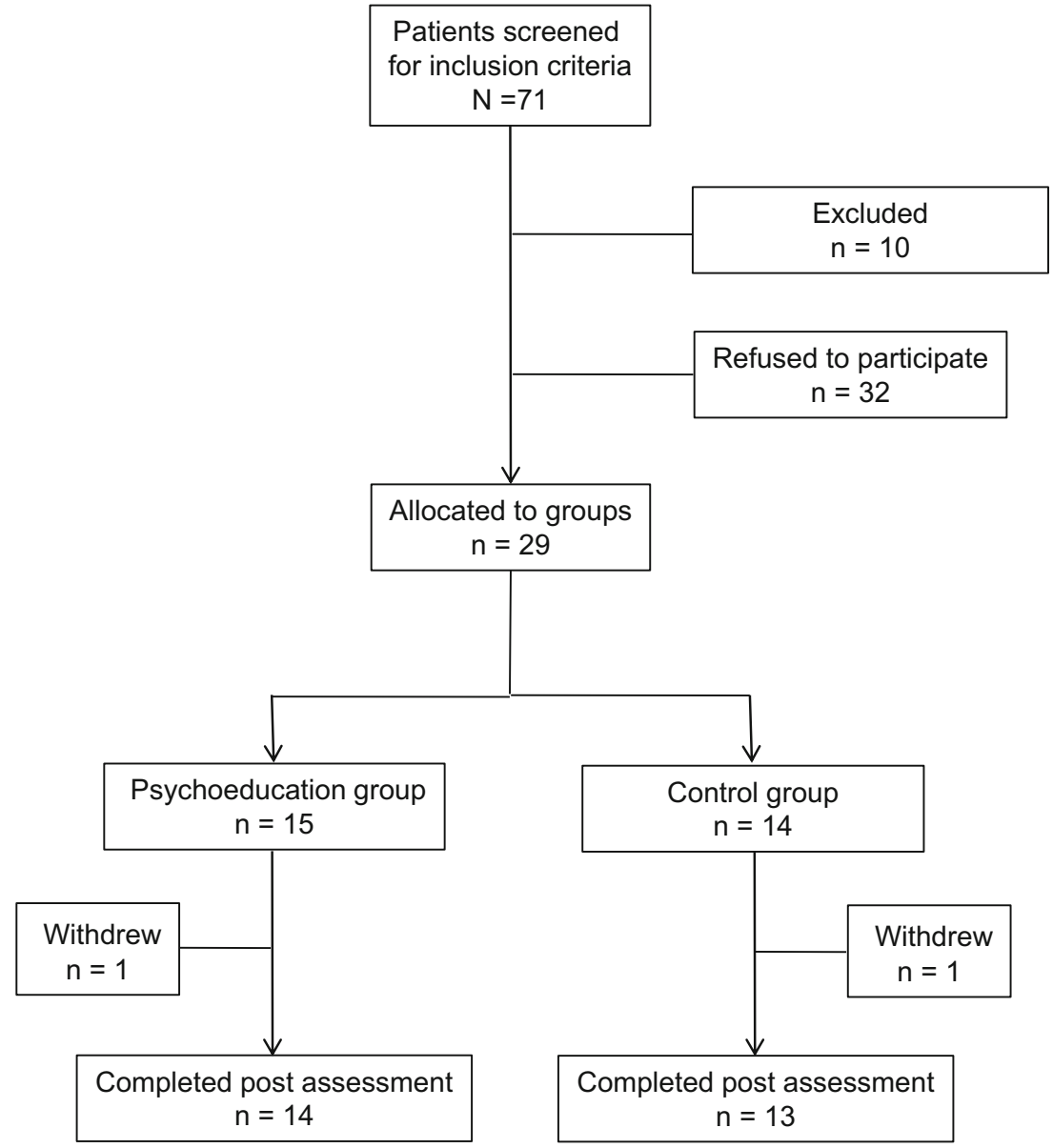

All statistical analysis was performed using the SPSS v.21. Analysis was performed per protocol using exact chi-square and linear-by-linear association statistics. Multiple regression analysis was conducted using the backward method. An alpha level of $p<.05$ and two tailed tests were used throughout the analysis.

\section{Sample and Clinical Characteristics at Baseline}

All data generated or analysed during this study are included in this published article (and its supplementary information files). Sixty-one patients met the inclusion criteria, of whom $48 \%(n=29)$ agreed to participate. Male patients accounted for $69 \%$ of the sample and female patients for $31 \%$; the same ratio as in the entire sample population. Participants' mean age was $40.93(S D=7.96)$ and approximately $83 \%$ of the participants had started pharmacotherapy after the age of $20(M=$ 23.21, $S D=6.41)$. More than half of the participants $(55 \%)$ had been in pharmacotherapy for over 15 years $(M=16.86$, $S D=9.58)$ and half $(55 \%)$ had been hospitalised one or two times $(M=1.93, S D=1.68)$, whereas no hospitalisation had occurred for approximately $17 \%$. The exact chi-square revealed no significant differences between the intervention and the control group in sex, and likewise, the independent sample t-test statistics indicated no significant differences between groups in any sociodemographic or clinical variable: age, marital status, number of people in the household, age that pharmacotherapy had started, years in pharmacotherapy and occurrences of hospitalisation. Likewise, the exact chisquare test revealed that there were no significant differences between the participants of the psychoeducation and the control group, in terms of the level of DAI, depression, anxiety and stress at baseline.

\section{Post-Measurements}

Analysis at post-intervention was performed per protocol including the 27 patients who completed the programme. Participants in the intervention group presented a significantly improved DAI level after psychoeducation, (pre: $M=0.53$, 
$S D=4.03$; post: $M=1.86, S D=5.23), X^{2}(1, N=27)=4.22$, $p=.04$, as well as a significantly reduced level of anxiety, (pre: $M=16.80, S D=15.00$; post: $M=15.14, S D=11.36$ ), $X^{2}(1, N=27)=3.57, p=.05$. Depression was increased (pre: $M=15.73, S D=7.70$; post: $M=18.29, S D=9.21$ ) but not at a significant level, $X^{2}(1, N=27)=1.11, p=.39$. Contrastingly to depression, stress level at post-intervention was increased at a significant level, (pre: $M=14.80, S D=$ 7.20; post: $M=14.86 ; S D=9.95), X^{2}(1, N=27)=8.52$, $p<.00$. The control group presented no significant change at post measurement of DAI level, (pre: $M=2.14, S D=4.40$; post: $M=2.46, S D=4.48), X^{2}(1, N=27)=1.32, p=.28$, depression (pre: $M=19.86, S D=9.65$; post: $M=18.31, S D=$ 6.58), $X^{2}(1, N=27)=0.32, p=.79$, anxiety (pre: $M=12.86$, $S D=7.00$; post: $M=10.15, S D=8.46), X^{2}(1, N=27)=0.53$, $p=.58$ and stress (pre: $M=19.14, S D=8.80$; post: $M=16.15$, $S D=6.95), X^{2}(1, N=27)=0.60, p=.52$.

Multiple regression analysis based on the backward procedure was conducted with dependent variable (DV) the level of adherence, and independent variables (IVs) the level of depression, anxiety, stress, occurrences of hospitalisation, years in pharmacotherapy, age pharmacotherapy started and number of people living in the household. The analysis was conducted twice, firstly with pre-post DAI and DASS variables as developed by subtracting pre-measurement scores from postmeasurement scores, and secondly, regression analysis was used separately for post-measurements (solely postpsychoeducation scores of DAI and DASS variables). Normal distribution of residuals and linearity could be assumed for all studied variables according to KolomogorovSmirnov test. Skewness and kurtosis of data was accepted as between -1.96 and 1.96 (Kim, 2013). There was no evidence of collinearity among the variables. In particular, the greatest value of Spearman's rho between the seven independent variables was found between post anxiety and post stress (with post level of adherence as the DV), $r_{s}=.73, p<.05$, and between pre-post anxiety and depression (with pre-post adherence as the DV), $r_{s}=.64, p<.05$. Collinearity diagnostics indicated that a very low level of multicollinearity was present for occurrences of hospitalisation (VIF $=1.00$, with DV prepost adherence and $V I F=1.54$, with DV post adherence). An acceptable level of multicolinearity was present for the remaining IVs in the model of the second regression (for years in pharmacotherapy $V I F=2.72$, tolerance 0.37 and for post level of depression $V I F=4.10$, tolerance 2.50 ). The first regression analysis indicated that pre-post improvement in DAI level of the psychoeducation group was significantly predicted only by occurrences of hospitalisation $(\beta=-0.60, p=.03)$, explaining $31 \%$ of the variance in DAI (adjusted $\mathrm{R}^{2}$ ), $R^{2}=$ $0.31, \mathrm{~F}(1,11)=6.26, p=.03$. All the remaining IVs, pre-post depression, pre-post anxiety, pre-post stress, years in pharmacotherapy, age pharmacotherapy started and number of people in the household were not found to be significant predictors of DAI change.

In the second regression model, patients' attitudes towards medication at post-intervention was significantly predicted by depression $(\beta=-1.07, p=.00)$, years in pharmacotherapy $(\beta=0.90, p=.01)$ and occurrences of hospitalisation $(\beta=$ $-0.83, p=.00$ and not by any other of the remaining variables of the model (post level of anxiety, age pharmacotherapy started, number of people in the household and post level of stress). The three predictors explained $65 \%$ of the variance in post DAI scores (Adjusted $\mathrm{R}^{2}$ ), $R^{2}=0.65, \mathrm{~F}(3,9)=8.37$, $p=.00$. Table 1 shows the results of the multiple regression for DAI level in the psychoeducation group at post measurement.

Table 1 Multiple regression for DAI level in the psychoeducation group at post measurement

\begin{tabular}{|c|c|c|c|c|c|c|c|c|c|c|c|c|c|c|c|}
\hline \multirow[t]{2}{*}{ Model } & \multicolumn{3}{|l|}{1} & \multicolumn{3}{|l|}{2} & \multicolumn{3}{|l|}{3} & \multicolumn{3}{|l|}{4} & \multicolumn{3}{|l|}{5} \\
\hline & $\beta$ & & $p$ & $\beta$ & & $p$ & $\beta$ & & $p$ & $\beta$ & & $p$ & $\beta$ & & $p$ \\
\hline Anxiety & 0.017 & & .971 & & & & & & & & & & & & \\
\hline $\begin{array}{l}\text { Age pharmacotherapy } \\
\text { started }\end{array}$ & -0.106 & & .700 & -0.105 & & .672 & & & & & & & & & \\
\hline People in the household & 0.227 & & .531 & 0.234 & & .390 & 0.290 & & & & & & & & \\
\hline Stress & -0.380 & & .453 & -0.365 & & .183 & -0.352 & & .165 & -0.448 & & .086 & & & \\
\hline Depression & -0.851 & & .068 & -0.853 & & $.041 *$ & -0.875 & & $.024 *$ & -0.718 & & $.044 *$ & -1074 & & $.004 * *$ \\
\hline Years in pharmacotherapy & 0.897 & & .080 & 0.903 & & $.036^{*}$ & 0.994 & & $.005 * *$ & 0.864 & & $.008 * *$ & 0.897 & & $.011 *$ \\
\hline $\begin{array}{l}\text { Occurences of } \\
\text { hospitalization }\end{array}$ & -0.577 & & .052 & -0.576 & & $.030 *$ & -0.588 & & .204 & -0.717 & & $.004 * *$ & -0.827 & & $.002 * *$ \\
\hline $\mathrm{F}$ & & 4580 & & & 6410 & & & 8642 & & & 9211 & & & 8372 & \\
\hline R2 (adjusted) & & 0.676 & & & 0.730 & & & 0.761 & & & 0.732 & & & 0.648 & \\
\hline
\end{tabular}

$D A I$, Drug Attitude Inventory

$* \mathrm{p}<.05, * * p<.01$ 


\section{Discussion}

Participants of both the psychoeducation and the control groups were chronically ill outpatients but not in an acute phase with a very low positive mean score of attitudes towards medication, at baseline. Also, the analysis of data at baseline indicated that on average patients had a moderate level of depression, severe anxiety and normal to mild stress. Research evidence suggests that adherence to medication tends to be improved among chronically ill patients (Linden and Schermuly-Haupt 2014). In the present study, approximately $55 \%$ of the participants reported that they had been in pharmacological treatment for 10 to 20 years and $31 \%$ for 20 to 35 years. However, despite the aforementioned characteristics (duration of illness, duration of pharmacotherapy and participants' membership at the day mental centre) both groups exhibited low positive attitudes towards medication at baseline. Indeed, research suggests that patients' mental health service engagement does not necessarily predict adherence (Lecomte et al. 2008; Tait et al. 2003), however, the severity of baseline anxiety in this sample of patients might partially explain low positive attitudes towards medication at baseline. As mentioned earlier, there is a lack of studies on the impact of anxiety on adherence to medication in schizophrenia, although according to previous research problematic adherence to medication is consistently associated with high neuroticism (Blackwell 1976).

Data analysis revealed that the two study groups did not differ significantly at baseline and that although the control group did not exhibit significant change, attitudes towards medication of the psychoeducation group were significantly improved at post-intervention measurement. Although the findings of this study are preliminary, they are consistent with previous research (Gonzalez-Soria et al. 2016; Pitschel-Walz et al. 2006). Interestingly, a meta-analysis (Xia et al. 2011), which included data from 44 psychoeducation RCTs, reports significant improvement in attitudes towards medication or adherence behaviour favouring both brief and longer psychoeducation programmes (Xia et al. 2011).

In addition, at post-intervention, it was found that a significant reduction in the level of anxiety of the psychoeducation group occurred, whereas the control group exhibited no significant change in any of the three variables. Furthermore, in the psychoeducation group, although depression was not increased at a significant level, stress level was found to be significantly increased after the intervention. This was unexpected and counter-intuitive; however, it may also be akin to the paradox of improvement, whereby patients' stress may increase short-term when they experience overall improvement in symptomatology. Worsening of stress has also been observed in studies on side-effects of psychotherapy (Klingberg et al. 2011; Linden and Schermuly-Haupt 2014) and may be interpreted in the light of research on insight
(Lincoln et al. 2007a, b; Palmer et al. 2015). An older metaanalysis (Pekkala and Merinder 2002) and its updated version (Xia et al. 2011) concluded that psychoeducation, although it significantly improves social and global functioning, quality of life, reduction in the number of relapses and duration of hospital admission, at the outset appears "off-putting" (Xia et al. 2011) to patients (p.28).

Thirdly, it was hypothesised that lower levels of depression, anxiety and stress would predict more positive attitudes towards medication. Regression analysis revealed mixed results. When regression was run at pre-post change level (prescores minus post-scores), findings indicated that the increase in positive attitudes towards medication was significantly predicted only by fewer occurrences of hospitalisation and not by depression, anxiety or stress. However, depression was found to be a significant predictor of attitudes towards medication at post-level analysis (solely post-psychoeducation scores), along with occurrences of hospitalisation and time in pharmacotherapy. The persisting significance of occurrences of hospitalisation found across statistical analyses is consistent with findings of other studies. For example, one study reports that fewer occurrences of hospitalisation along with the quality of interpersonal relationships and severity of symptoms predicted better attitudes towards medication (García-Cabeza et al. 2000). In addition, an RCT psychoeducation study reports significantly fewer relapses for the psychoeducation group (compared to the standard care group), and specifically for those patients with fewer hospitalisations in the past (Pitschel-Walz et al. 2006).

Research suggests that occurrences of hospitalisation affect patients' feelings of stigmatisation. A study reports that $64 \%$ of the 127 participants believed that occurrences of hospitalisation are perceived by others as "failure" (Kleim et al. 2008, p. 486). In those terms, psychoeducational interventions with the objective of destigmatising medication intake might be particularly beneficial for patients with longer clinical history. Patients who internalise stigma have feelings of shame, loss and entrapment related to illness, which increase their vulnerability to depression (Birchwood et al. 2006). In the current study stigma was not measured; however, it was observed that patients with more occurrences of hospitalisation had greater improvement in anxiety and kept stress stable or relatively stable. Nevertheless, with regard to attitudes towards medication, the small sample size possibly did not allow for detection of patterns relating these variables (improvement in anxiety and stability of stress) to the significant improvement in attitudes towards medication.

\section{Limitations}

This was a pilot study and has several limitations. The sample size was small and entirely comprised by patients in remission phase, who were attendants of a day mental health centre. 
Therefore the findings should be treated as preliminary; generalisation of results is not possible and interpretations entailed should be seen tentatively. Data analysis was performed per protocol (excluding one patient from the intervention group and one patient from the control group, who did not complete the study), decreasing the sample even more. However, statistical power issues are overcome with the use of exact tests, where $p$-values are accurately computed.

Furthermore, in terms of the sample characteristics, some researchers suggest that being in remission phase - with few or no psychotic symptoms - might negatively influence attitudes towards medication, as patients may feel they are in no need of medication (Kane et al. 2013; Marder 2003). Following this rationale, the significant improvement of patients' attitudes towards medication could be partially attributed to low scores of patients at baseline. Similarly, the improvement of anxiety at post-psychoeducation might be due to elevated anxiety levels at baseline. However, the control group exhibited similar scores at baseline (and across all variables). In addition, the partial attendance to psychoeducation might have further influenced the results. In terms of possible bias, the two study groups were found similar at baseline in regard to clinical and demographic characteristics as well as all other measured variables, with no needed correction. Also, during invitation to participate, patients were briefed on what their participation would entail whether they were placed in the psychoeducation or the control group and were randomized to study groups after the completion of recruiting process, thereby eliminating self-selection bias. In addition, pre-post attitudes towards medication, was in some cases radically changed towards a negative direction after the intervention, indicating that disclosure of information was reasonably unbiased in the psychoeducation group.

In a future replication it would be meaningful to include comparison to a second psychoeducational intervention along with a control group and measuring self-stigma and insight into illness (along with depression, anxiety and stress), with collection of follow-up data. Also, collection of additional information for patients' characteristics, such as information about their prior experience with antipsychotic medication, treatment response, side effects, tolerance etc., would be fruitful for further analysis of the participants' attitudes towards medication and effects of the current hope-based psychoeducation.

\section{Implications and Future Research}

There are several implications for research and clinical practice. A plausible explanation of the mixed effects of depression on medication adherence might stem from the interaction of stigma with insight into illness. Based on the varying evidence, insight is not dysfunctional only when it entails poor adherence (Beck et al. 2011). A hypothesis that might be proved significant in terms of predicting adherence, is that insight is rather dysfunctional when it entails an increase in self-stigma (Nordick and van Heugten 2014) or passivity/ withdrawal (Fleischhaker et al., 2003; Roberts and Wolfson 2004), with either poor or good adherence. Hope-based psychoeducation could help patients to de-stigmatise the use of medication, while targeting patients' illness-related passivity and fostering improvement of insight into illness.

Patients' insight into illness differs from person to person and may also change within a patient, from time to time, as insight into illness can be influenced by changes of the clinical and situational factors over time (Donohoe et al. 2001). Also, there is some evidence that patients' increasing insight into illness might not only intensify depression but also raise the reaction to stigma and consequently to pharmacotherapy (Nordick and van Heugten 2014). There has been evidence that being in the form of a seminar psychoeducation can offer a stable knowledge base to patients that protects them from fluctuations of insight into illness (Al-Yahya 2014; Ruzanna et al. 2010), negative attitudes towards medication (Choe et al. 2015) and spasmodic reactance to pharmacotherapy or insufficient adherence into medication over time (Pitschel-Walz et al. 2006).

Moreover, patients' self-stigmatisation and negativity is apparent in depression and in increased insight and is also closely related to negative symptoms (Rector et al. 2005). Therefore, it is also important that psychoeducation in schizophrenia include sufficient information about the hormonebiological linkages between anxiety, depression, dietary intake and exercise. This information is needed by patients not only because they are at risk of chronic health conditions (Wearden 2014), but also because such information helps patients to be able to recognize early signs of relapse, improve their coping and also their sense of agency.

Follow up data is needed in future research to measure effects of hope-based psychoeducation on these markers of patients' quality of life and illness course - as are depression, stress, anxiety and adherence - with follow-up data. Also, it would be valuable to test differences of hope-based psychoeducation effects on depressive and anxiety symptoms between diverse patient groups in terms of illness-course chronicity and severity. In addition, there is a need for research on the effects of stress, anxiety and depressive symptomatology in relation to adherence in schizophrenia in general and in relation to stigma and insight into illness.

\section{Conclusion}

Psychoeducation can be effective in addressing a wide range of illness-related issues that compound schizophrenia's course, such as depression, anxiety, stress, stigma, insight into illness and attitudes towards medication. Research suggests that psychosocial symptoms, as well as their impact on 
adherence to medication, are qualitatively diversified by stigma and insight, while they are also subject to individual, clinical and situational factors. The preliminary results of this study suggest that a hope-based psychoeducational intervention, with cautious communication of causal models and the aim to empower patients' knowledge and sense of agency, could possibly contribute to positive attitudes towards medication and potentially alleviate clinical symptoms.

\section{Compliance with Ethical Standards}

Ethical Approval All procedures performed in studies involving human participants were in accordance with the ethical standards of the institutional and/or national research committee and with the 1964 Declaration of Helsinki and its later amendments or comparable ethical standards.

Informed Consent Informed consent was obtained from all individual participants included in the study.

Conflict of Interest The authors declare that they have no conflict of interest.

Open Access This article is distributed under the terms of the Creative Commons Attribution 4.0 International License (http:// creativecommons.org/licenses/by/4.0/), which permits unrestricted use, distribution, and reproduction in any medium, provided you give appropriate credit to the original author(s) and the source, provide a link to the Creative Commons license, and indicate if changes were made.

\section{References}

AlAqeel, B., \& Margolese, H. C. (2012). Remission in schizophrenia: Critical and systematic review. Harvard Review of Psychiatry, 20(6), 281-297. https://doi.org/10.3109/10673229.2012.747804.

Al-Jeshi, A.A., Epstein, I., \& Zipursky, R. B. (2006). An overview of the genetic risk of developing schizophrenia in relatives of schizophrenic patients. Neurosciences, 11(1), 7-10. PMID: 22266495.

Al-Yahya, N. M. (2014). Effects of psycho education intervention in improving insight and medication compliance of schizophrenic clients, Riyadh, Saudi Arabia. World Journal of Medical Sciences, 11(3), 289-300. https://doi.org/10.5829/idosi.wjms.2014.11.3. 84256.

Atkinson, J. M., Coia, D. A., Gilmour, W. H., \& Harper, J. P. (1996). The impact of education groups for people with schizophrenia on social functioning and quality of life. British Journal of Psychiatry, 168(2), 199-204. https://doi.org/10.1192/bjp.168.2.199.

Baüml, J., Froböse, T., Kraemer, S., Rentrop, M., \& Pitschel-Walz, G. (2006). Psychoeducation: A basic psychotherapeutic intervention for patients with schizophrenia and their families. Schizophrenia Bulletin, 32(1), 1-9. https://doi.org/10.1093/schbul/sbl017.

Beck, E. M., Cavelti, M., Wirtz, M., Kossowsky, J., \& Vauth, R. (2011). How do socio-demographic and clinical factors interact with adherence attitude profiles in schizophrenia? A cluster-analytical approach. Psychiatry Research, 187(1-2), 55-61. https://doi.org/10. 1016/j.psychres.2010.10.012.

Birchwood, M., Mason, R., Macmillan, F., \& Healey, J. (1993). Depression, demoralization and control over psychotic illness: A comparison of depressed and non-depressed patients with a chronic psychosis. Psychological Medicine, 23(2), 387-395. https://doi.org/ 10.1017/S0033291700028488.

Birchwood, M., Trower, P., Brunet, K., Gilbert, P., Iqbal, Z., \& Jackson, C. (2006). Social anxiety and the shame of psychosis: A study in first episode psychosis. Behaviour Research and Therapy, 45(5), 1025-1037. https://doi.org/10.1016/j.brat.2006.07.011.

Blackwell, B. (1976). Treatment adherence. British Journal of Psyhciatry, 129, 513-521. https://doi.org/10.1192/bjp.129.6.513.

Blelch, A., Brown, S. L., Kahn, R., \& van Praag, H. M. (1988). The role of serotonin in schizophrenia. Schizophrenia Bulletin, 14(2), 297315. PMID: 3059473.

Bonney, S., \& Stickley, L. (2008). Recovery and mental health review: A review of the British literature. Journal of Psychiatric \& Mental Health nurse, 15(2), 140-153. https://doi.org/10.1111/j.1365-2850. 2007.01185.x.

Bottonari, K. A., Roberts, J. E., Ciesla, J. A., \& Hewitt, R. G. (2005). Life stress and adherence to antiretroviral therapy among hiv-positive individuals: A preliminary investigation. AIDS Patient Care and STDs, 19(11), 719-727. https://doi.org/10.1089/apc.2005.19.719.

Bowirrat, A., Chen, T. J., Blum, K., Madigan, M., Bailey, J., Chen, A. L., et al. (2010). Neuro-psychopharmacogenetics and neurological antecedents of posttraumatic stress disorder: Unlocking the mysteries of resilience and vulnerability. Current Neuropharmacology, 8(4), 335-358. https://doi.org/10.2174/157015910793358123.

de Brito, D. C., Marsicano, E. O., dos Santos Grincenkov, F. R., Colugnati, F. A., Lucchetti, G., \& Sanders-Pinheiro, H. (2016). Stress, coping and adherence to immunosuppressive medications in kidney transplantation : A comparative study. Sao Paolo Medical Journal, 134(4), 292-299. https://doi.org/10.1590/15163180.2015.01071008.

Brown, A. S. (1976). The environment and susceptibility to schizophrenia. International Journal of Health Services, 6(3), 521-538. https:// doi.org/10.1016/j.pneurobio.2010.09.003.

Browne, S., Roe, M., Lane, A., \& O'Callaghan, E. (1996). A preliminary report on the effect of a psychosocial and educative rehabilitation programme on quality of life and symptomatology in schizophrenia. European Psychiatry, 11(8), 386-389. https://doi.org/10.1016/ S0924-9338(97)82574-6.

Buckley, P. F., Miller, B. J., Lehrer, D. S., \& Castle, D. J. (2009). Psychiatric comorbidities and schizophrenia. Schizophrenia Bulletin, 35(2), 383-402. https://doi.org/10.1093/schbul/sbn135.

Choe, K., Sung, B. J., Kang, Y., \& Yoo, S. Y. (2015). Impact of psychoeducation on knowledge of and attitude toward medications in clients with schizophrenia and schizoaffective disorders. Perspectives in Psychiatric Care, 52(2), 113-119. https://doi.org/ 10.1111/ppc.12106.

Conti, V., Lora, A., Cipriani, A., Fortino, I., Merlino, L., \& Barbui, C. (2012). Persistence with pharmacological treatment in the specialist mental healthcare of patients with severe mental disorders. European Journal of Pharmacology, 68(12), 1647-1655. https:// doi.org/10.1007/s00228-012-1298-2.

Corrigan, W. P., \& Rao, D. (2012). On the self-stigma of mental illness: Stages, disclosure, and strategies for change. The Canadian Journal of Psychiatry, 57(8), 464-469. https://doi.org/10.1177/ 070674371205700804.

Cullen, B. A., McGinty, E. E., Zhang, Y., dosReis, S. C., Steinwachs, D. M., Guallar, E., \& Daumit, G. L. (2013). Guideline-concordant antipsychotic use and mortality in schizophrenia. Schizophrenia Bulletin, 39(5), 1159-1168. https://doi.org/10.1093/schbul/sbs097.

Czeh, B., \& Lucassen, P. J. (2007). What causes the hippocampal volume decrease in depression? Are neurogenesis, glial changes and apoptosis implicated? European Archives of Psychiatry and Clinical Neuroscience, 257(5), 250-260. https://doi.org/10.1007/s00406007-0728-0s.

DeLisi, L. E., Shaw, S. H., Crow, T. J., Shields, G., Smith, A. B., Larach, V. W., Wellman, N., Loftus, J., Nanthakumar, B., Razi, K., Stewart, 
J., Comazzi, M., Vita, A., Heffner, T., \& Sherrington, R. (2002). A genome-wide scan for linkage to chromosomal regions in $382 \mathrm{sib}-$ ling pairs with schizophrenia or schizoaffective disorder. The American Journal of Psychiatry, 159(5), 803-812. https://doi.org/ 10.1176/appi.ajp.159.5.803.

DeQuardo, J. R., Tandon, R., Goldman, R., Meador-Woodruff, J. H., McGrath-Giroux, M., Brunberg, J. A., \& Kim, L. (1994). Ventricular enlargement, neuropsychological status and premorbid function in schizophrenia. Biological Psychiatry, 35(8), 517-524. https://doi.org/10.1016/0006-3223(94)90098-1.

DiMatteo, M. R., Lepper, H. S., \& Croghan, T. W. (2000). Depression is a risk factor for noncompliance with medical treatment: Meta-analysis of the effects of anxiety and depression on patient adherence. Archives of Internal Medicine Journal, 160(14), 2101-2107. https://doi.org/10.1001/archinte.160.14.2101.

Donohoe, G., Owens, N., O’Donnell, C., Burke, T., Moore, L., Tobin, A., \& O'Callaghan, E. (2001). Predictors of compliance with neuroleptic medication among inpatients with schizophrenia: A discriminant function analysis. European Psychiatry, 16(5), 293-298. https://doi. org/10.1016/S0924-9338(01)00581-8.

Eisner, E., Drake, R., \& Barrowclough, C. (2013). Assessing early signs of relapse in psychosis: Review and future directions. Clinical Psychology Review, 33(5), 637-653. https://doi.org/10.1016/j.cpr. 2013.04.001

Farrell, S. P., Hains, A. A., Davies, W. H., Smith, P., \& Parton, E. (2004). The impact of cognitive distortions, stress and adherence on metabolic control in youths with type 1 diabetes. Journal of Adolescent Health, 34(6), 461-467. https://doi.org/10.1016/S1054-139X(03) 00215-5.

Fleischhacker, W. W., Oehl, M. A., \& Hummer, M. (2003). Factors influencing compliance in schizophrenia patients. Journal of Clinical Psychiatry, 64(16), 10-13 Retrieved from https://www. ncbi.nlm.nih.gov/pubmed/14680413. Accessed 8 Dec 2017.

García-Cabeza, I., Sanz Amador, M., ArangoLópez, C., \& González de Chávez, M. (2000). Subjective response to antipsychotics in schizophrenic patients: Clinical implications and related factors. Schizophrenia Research, 41(2), 349-355. https://doi.org/10.1016/ S0920-9964(99)00079-1.

Gaur, N., Gautam, S., Gaur, M., Sharma, P., Dadheech, G., \& Mishra, S. (2008). The biochemical womb of schizophrenia: A review. Indian Journal of Clinical Biochemistry, 23(4), 307-327. https://doi.org/ 10.1007/s12291-008-0071-x.

Giráldez, S. L., Fernández, O. V., Iglesias, P. F., Pedrero, E. F., \& Paino, M. (2011). New trends in treatment for psychosis. Psychology in Spain, 15(1), 33-47 Retrieved from http://www. psychologyinspain.com/content/full/2011/15004.pdf. Accessed 26 Nov 2017.

Gonzalez, J. S., Peyrot, M., McCarl, L. A., Collins, E. M., Serpa, L., Mimiaga, M. J., \& Safren, S. A. (2008). Depression and diabetes treatment non adherence: A meta-analysis. Diabetes Care, 31(12), 2398-2403. https://doi.org/10.2337/dc08-1341.

Gonzalez-Soria, C., Fombellida-Velasco, C. I., Fernández-Martín, L. C., Sanchez-Pernas, L. S., Al Chaal-Marcos, L., et al. (2016). Attitude toward medication as a predictor of therapeutic adherence [abstract]. Importance of psychoeducation on treatment. European Psychiatry, 33, S565-S566. https://doi.org/10.1016/j.eurpsy.2016.01.2096.

Grenard, J. L., Munjas, B. A., Adams, J. L., Suttorp, M., Maglione, M., McGlynn, E. A., \& Gellad, W. (2011). Depression and medication adherence in the treatment of chronic diseases in the United States: A meta-analysis. Journal of General Internal Medicine, 26(10), 1175-1182. https://doi.org/10.1007/s11606-011-1704-y.

Guillin, O., \& Laruelle, M. (2005). Neurobiology of dopamine in schizophrenia. Cellscience, 2(2). https://doi.org/10.1016/S0074-7742(06) 78001-1.

Haddad, P. M., Brain, C., \& Scott, J. (2014). Non adherence with antipsychotic medication in schizophrenia : Challenges and management strategies. Dove Press, 5, 43-62. https://doi.org/10. 2147/PROM.S42735.

Hans, S. L., Auerbach, J. Q., Styr, B., \& Marcus, J. (2004). Offspring of parents with schizophrenia: Mental disorders during childhood and adolescence. Schizophrenia Bulletin, 30(2), 303-315. https://doi. org/10.1093/oxfordjournals.schbul.a007080.

Harding, C. M. (1988). Course types in schizophrenia: An analysis of European and American studies. Schizophrenia Bulletin, 14(4), 633-643. https://doi.org/10.1093/schbul/14.4.633.

Herz, M. I., Lamberti, J. S., Mintz, J., Scott, R., O’Dell, S. P., McCartan, L., \& Nix, G. (2000). A program for relapse prevention in schizophrenia: A controlled study. Archives of General Psychiatry, 57(3), 277-283. https://doi.org/10.1001/archpsyc.57.3.277.

Higashi, K., Medic, G., Littlewood, K. J., Diez, T., Granström, O., \& De Hert, M. (2013). Medication adherence in schizophrenia: Factors influencing adherence and consequences of non adherence, a systematic literature review. Therapeutic Advances in Psychopharmacology, 3(4), 200-218. https://doi.org/10.1177/ 2045125312474019.

Hoffmann, T. C. (2014). et al., Better reporting of interventions: Template for intervention description and replication (TIDieR) checklist and guide. British Medical Journal, 348(1687). https://doi.org/10.1136/ bmj.g1687.

Hogan, T. P., Awad, A. G., \& Eastwood, K. (1983). A self-report scale predictive of drug compliance in schizophrenics: Reliability and discriminative ability. Psychological Medicine, 13, 177-183. https://doi.org/10.1017/S0033291700050182.

Hornung, W. P., Klingberg, S., Feldmann, R., Schonauer, K., \& Schulze Mönking, H. (1998). Collaboration with drug treatment by schizophrenic patients with and without psychoeducational training: Results of a 1-year follow-up. Acta Psychiatrica Scandinavica, 97(3), 213-219. https://doi.org/10.1111/j.1600-0447.1998.tb09990.

Huppert, J. D., Weiss, K. A., Lim, R., Pratt, S., \& Smith, T. E. (2001). Quality of life in schizophrenia: Contributions of anxiety and depression. Schizophrenia Research, 52(2-3), 171-180. https://doi. org/10.1016/S0920-9964(99)00151-6.

Jimmy, B., \& Jose, J. (2011). Patient medication adherence: Measures in daily practice. Oman Medical Journal, 26(3), 155-159. https://doi. org/10.5001/omj.2011.38.

Kane, J. M., Kishimoto, T., \& Correll, C. U. (2013). Non-adherence to medication in patients with psychotic disorders: Epidemiology, contributing factors and management strategies. World Psychiatry, 12(3), 216-226. https://doi.org/10.1002/wps.20060.

Kim, H.Y. (2013). Statistical notes for clinical researchers: assessingnormal distribution (2) using skewness and kurtosis. Restorative Dentistry and Endodontics, 2234-7658. https://doi.org/ 10.5395/rde.2013.38.1.52.

Kleim, B., Vauth, R., Adam, G., Stieglitz, R., Hayward, P., \& Corrigan, P. (2008). Perceived stigma predicts low self-efficacy and poor coping in schizophrenia. Journal of Mental Health, 17(5), 482-491. https:// doi.org/10.1080/09638230701506283.

Klingberg, S., Wölwer, W., Engel, C., Wittorf, A., Herrlich, J., Meisner, C., Buchkremer, G., \& Wiedemann, G. (2011). Negative symptoms of schizophrenia as primary target of cognitive behavioral therapy: Results of the randomized clinical TONES study. Schizophrenia Bulletin, 37(2), 98-110. https://doi.org/10.1093/schbul/sbr073.

Lecomte, T., Spidel, A., Leclerc, C., MacEwan, G. W., Greaves, C., \& Bentall, R. P. (2008). Predictors and profiles of treatment nonadherence and engagement in services problems in early psychosis. Schizophrenia Research, 102, 295-302. https://doi.org/10.1016/j. schres.2008.01.024.

Li, X., Huang, L., Wang, H., Fennie, H. G., \& Williams, A. B. (2011). Stigma mediates the relationship between self-efficacy, medication adherence, and quality of life among people living with hiv/aids in 
China. AIDS Patient Care, 25(11), 665-671. https://doi.org/10. 1089/apc.2011.0174.

Lincoln, T. M., Lüllmanm, E., \& Rief, W. (2007a). Correlates and longterm consequences of poor insight in patients with schizophrenia, a systematic review. Schizophrenia Bulletin, 33(6), 1324-1342. https://doi.org/10.1093/schbul/sbm002.

Lincoln, T. M., Wilhelm, K., \& Nestoriuc, Y. (2007b). Effectiveness of psychoeducation for relapse, symptoms, knowledge, adherence and functioning in psychotic disorders: A meta-analysis. Schizophrenia Research, 96(1-3), 232-245. https://doi.org/10.1016/j.schres.2007. 07.022

Linden, M., \& Schermuly-Haupt, M. L. (2014). Definition, assessment and rate of psychotherapy side effects. World Psychiatry, 13(3), 306-309. https://doi.org/10.1002/wps.20153.

Lopresti, A. L., Hood, S. D., \& Drummond, P. D. (2013). A review of lifestyle factors that contribute to important pathways associated with major depression: Diet, sleep and exercise. Journal of Affective Disorders, 148(1), 12-27. https://doi.org/10.1016/j.jad. 2013.01.014.

Lovibond, P. H., \& Lovibond, S. H. (1995). The structure of negative emotional states: Comparison of the depression anxiety stress scales (DASS) with the Beck depression and anxiety inventories. Behaviour Research and Therapy, 33(3), 335-343. https://doi.org/ 10.1016/0005-7967(94)00075-U.

Lüllmann, E., Berendes, S., Rief, W., \& Lincoln, T. M. (2011). Benefits and harms of providing biological causal models in the treatment of psychosis: An experimental study. Journal of Behavior Therapy and Experimental Psychiatry, 42(4), 447-453. https://doi.org/10.1016/j. jbtep.2011.03.003.

Lyrakos, G. N., Arvaniti, C., Smyrnioti, M., \& Kostopanagiotou, G. (2011). Translation and validation study of the depression anxiety stress scale in the greek general population and in a psychiatric patient's sample. European Psychiatry, 26(1), 1731. https://doi.org/ 10.1016/S0924-9338(11)73435-6.

Lyrakos, G., Hatziagelaki, E., Spinaris, B., Damigos, D., Spyropoulos, I., \& Kostopanagiotou, G. (2012). The comparison of fatigue between patients with diabetes melittus, psychiatric outpatients and general population in Greece. European Psychiatry, 27(1), 1. https://doi.org/ 10.1016/S0924-9338(12)74399-7.

Lyrakos, G., Spyropoulos, I., \& Spinaris, V. (2014). Factors affecting compliance with therapy in psychiatric inpatients during treatment. European Psychiatry, 29(1), 1. https://doi.org/10.1016/S09249338(14)78186-6.

Lysaker, P. H., \& Buck, K. D. (2008). Is recovery from schizophrenia possible? An overview of concepts, evidence, and clinical implications. Primary Psychiatry, 15, 60-65 Retrieved from http:// primarypsychiatry.com. Accessed 13 Feb 2016.

Lysaker, P. H., Roe, D., \& Yanos, P. T. (2007). Toward understanding the insight paradox : Internalized stigma moderates the association between insight and social functioning, hope and self-esteem among people with schizophrenia spectrum disorders. Schizophrenia Bulletin, 33(1), 192-199. https://doi.org/10.1093/schbul/sbl016.

Marder, S. R. (2003). Overview of partial compliance. Journal of Clinical Psychiatry, 64(16), 3-9. PMID: 14680412.

Martos-Mendez, M. J. (2015). Self-efficacy and adherence to treatment: The mediating effects of social support. Journal of Behavior, Health \& Social Issues, 7(2), 19-29. https://doi.org/10.5460/jbhsi.v7.2. 52889.

Morgan, V., Castle, D., Page, A., Fazio, S., Gurrin, L., Burton, P., Montgomery, P., \& Jablensky, A. (1997). Influenza epidemics and incidence of schizophrenia, affective disorders and mental retardation in Western Australia: No evidence of a major effect. Schizophrenia Bulletin, 26, 25-39. https://doi.org/10.1016/S09209964(97)00033-9.

Na, E., Yim, S. J., Lee, J. N., Kim, J. M., Hong, K., Hong, M. H., \& Han, H. (2015). Relationships among medication adherence, insight and neurocognition in chronic schizophrenia. Psychiatric and Clinical Neurosciences, 69(5), 298-304. https://doi.org/10.1111/pcn.12272.

National Institute for Clinical Excellence (2014). Psychosis and schizophrenia in adults (Update). NICE Clinical Guideline 178. Available at www.nice.org.uk/cg178. Accessed 10 Sep. 2017.

Nordick, W., \& van Heugten, K. (2014). Insight development in schizophrenia. The construction of dangerousness in relapse. Qualitative Health Research, 24(5), 591-602. https://doi.org/10.1177/ 1049732314529023.

Opler, M., Charap, J., Greig, A., Stein, V., Polito, S., \& Malaspina, D. (2013). Environmental risk factors and schizophrenia. International Journal of Mental Health, 42(1), 23-32. https://doi.org/10.2753/ IMH0020-7411420102.

Palmer, E. C., Gileen, J., \& David, A. S. (2015). The relationship between cognitive insight and depression in psychosis and schizophrenia: A review and meta-analysis. Schizophrenia Research, 166(1-3), 261268. https://doi.org/10.1016/j.schres.2015.05.032.

Pekkala, E. T., \& Merinder, L. B. (2002). Psychoeducation for schizophrenia. Cochrane Database of Systematic Reviews, 2, CD002831. https://doi.org/10.1002/14651858.CD002831.

Perkins, D. O., Johnson, J. L., Hamer, R. M., Zipursky, R. B., Keefe, R. S., Centorrhino, F., et al. (2006). Predictors of antipsychotic medication adherence in patients recovering from a first psychotic episode. Schizophrenia Research, 83, 53-63. https://doi.org/10.1016/j. schres.2005.10.016.

Perkins, D. O., Gu, H., Weiden, P. J., McEvoy, J. P., Hamer, R. M., \& Lieberman, J. A. (2008). Predictors of treatment discontinuation and medication nonadherence in patients recovering from a first episode of schizophrenia, schizophreniform disorder, or schizoaffective disorder: A randomized, double-blind, flexible-dose, multicenter study. Journal of Clinical Psychiatry, 69(1), 106-113. PMID: 18312044.

Pitschel-Walz, G., Bäuml, J., Bender, W., Engel, R. R., Wagner, M., \& Kissling, W. (2006). Psychoeducation and compliance in the treatment of schizophrenia: Results of the Munich psychosis information project study [abstract]. The Journal of Clinical Psychiatry, 67(3), 443-452. https://doi.org/10.4088/JCP.v67n0316.

Read, J., Haslam, N., Sayce, L., \& Davies, E. (2006). Prejudice and schizophrenia: A review of the 'mental illness is an illness like any other' approach. Acta Psychiatrica Scandinavica, 114(5), 303-318. https://doi.org/10.1111/j.1600-0447.2006.00824.x.

Rector, N., Beck, A. T., \& Stolar, N. (2005). The negative symptoms of schizophrenia: A cognitive perspective. Canadian Journal of Psychiatry, 50(5), 247-257. https://doi.org/10.3410/f.13853959. 15292059

Roberts, G., \& Wolfson, P. (2004). The rediscovery: Open to all. Advances in Psychiatric Treatment, 10, 37-48. https://doi.org/10. 1192/apt.10.1.37.

Ruzanna, Z., Marhani, M., Parveen, K., \& Cheah, Y. C. (2010). Does psychoeducation improve insight of patients with schizophrenia? Malaysian Journal of Psychiatry, 19, 27-40 Retrieved from http:// mjpsychiatry.org/index.php/mjp/article/viewFile/97/89. Accessed 11 Aug 2018.

Santone, G., Rucci, P., Lazzarotto, M., Monaci, A., Ciarafoni, C., \& Borsetti, G. (2008). Attitudes toward medication in inpatients with schizophrenia : A cluster analytic approach. Psychiatry Research, 158(3), 324-334. https://doi.org/10.1016/j.psychres.2006.12.010.

Sapra, M., Vahia, I. V., Reyes, P. N., Ramirez, P., \& Cohen, C. I. (2008). Subjective reasons for adherence to psychotropic medication and associated factors among older adults with schizophrenia. Schizophrenia Research, 106, 348-355. https://doi.org/10.1016/j. schres.2008.09.004.

Schennach-Wolff, R., Jäger, M., Seemuller, F., Obermeier, M., Messer, T., Laux, G., et al. (2009). Attitude towards adherence in patients with schizophrenia at discharge. Journal of Psychiatric Research, 43(16), 1294-1301. https://doi.org/10.1016/j.jpsychires.2009.05.003. 
Schrank, B., Stanghellini, G., \& Slade, M. (2008). Hope in psychiatry: A review of the literature. Acta Psychiatrica Scandinavica, 118(6), 421-433. https://doi.org/10.1111/j.1600-0447.2008.01271.x.

Staring, A. B. P., Mulder, C. L., Duivenvoorden, H. J., De Haan, L., \& Van der Gaag, M. (2009). Fewer symptoms vs. more side-effects in schizophrenia? Opposing pathways between antipsychotic medication compliance and quality of life. Schizophrenia Research, 113, 27-33. https://doi.org/10.1016/j.schres.2009.05.022.

Steger, K. A., Cassidy, C., Rabinovitch, M., Joober, R., \& Malla, A. (2012). Impact of symptom resolution on medication adherence in fi rst episode psychosis. Psychiatry Research, 196(1), 45-51. https:// doi.org/10.1016/j.psychres.2011.10.015.

Tait, L., Birchwood, M., \& Trower, D. (2003). Predicting engagement with services for psychosis: Insight, symptoms and recovery style. British Journal of Psychiatry, 182, 123-128. https://doi.org/10. 1192/bjp.182.2.123.

Tienari, P., Sorri, A., Lahti, I., Naarala, M., Wahlberg, K. E., Moring, J., ... Wynne, L. C. (1987). Genetic and psychosocial factors in schizophrenia: The Finnish adoptive family study. Schizophrenia Bulletin, 13(3), 477-484. PMID: 3629201.

Tienari, P., Wynne, L. C., Moring, J., Läsky, K., Nieminen, P., Sorri, A., et al. (2000). Finnish adoptive family study: Sample selection and adoptee DSM-III-R diagnoses. Acta Psychiatrica Scandinavica, 101(6), 433-443. https://doi.org/10.1034/j.1600-0447.2000. 101006433.x.

Tienari, P., Wynne, L. C., Läsky, L., Moring, J., Nieminen, P., Sorri, A., et al. (2003). Genetic boundaries of the schizophrenia spectrum: Evidence from the Finnish adoptive family study of schizophrenia. The American Journal of Psychiatry, 160(9), 1587-1594. https:// doi.org/10.1176/appi.ajp.160.9.1587.

Üçok, A., Polat, A., Çakır, S., \& Genç, A. (2006). One year outcome in first episode schizophrenia: Predictors of relapse. European
Archives of Psychiatry and Clinical Neuroscience, 256(1), 37-43. https://doi.org/10.1007/s00406-005-0598-2.

Ward, D. B. (2014). The process and implications of self-stigma in schizophrenia. Concept, 37. Retrieved from https://concept. journals. Accessed 16 June 2018.

Wearden, A. (2014). Health behaviour interventions should not neglect people with serious mental health problems. British Journal of Health Psychology, 19(4), 683-687. https://doi.org/10.1111/bjhp. 12117.

World Health Organization (2003). Adherence to long term therapies. Evidence for action. Retrieved from: http://www.who.int/chp/ knowledge/publications/adherence_full_report.pdf?ua=1. Accessed 21 Jan. 2016.

Xia, J., Merinder L. B., \& Belgamwar, M. R. (2011). Psychoeducation for schizophrenia. Cochrane Database of Systematic Reviews, 6, CD002831. https://doi.org/10.1002/14651858.CD002831.pub2.

Yang, J., Ko, Y., Paik, J., Lee, M., Han, C., Joe, S., et al. (2012). Symptom severity and attitudes toward medication : Impacts on adherence in outpatients with schizophrenia. Schizophrenia Research, 134(2-3), 226-231. https://doi.org/10.1016/j.schres.2011.11.008.

Zipursky, R. B., Reilly, T., \& Murray, R. M. (2012). The myth of schizophrenia as a progressive brain disease. Schizophrenia Bulletin, 39(6), 1363-1372. https://doi.org/10.1093/schbul/sbs135.

Züchner, S., Roberts, S. T., Speer, M. C., \& Beckham, J. C. (2007). Update on psychiatric genetics. Genetics in Medicine, 9(6), 332 340. https://doi.org/10.1097/GIM.0b013e318065a9fa.

Publisher's note Springer Nature remains neutral with regard to jurisdictional claims in published maps and institutional affiliations. 